\title{
Simplemente: el plano inclinado
}

(Simply, the incline)

\author{
C.H. Wörner \\ Instituto de Física, Pontificia Universidad Católica de Valparaíso, Valparaíso, Chile
}

Recebido em 18/1/2012; Aceito em 23/4/2012; Publicado em 6/2/2012

\begin{abstract}
Se describen algunos montajes experimentales simples de planos inclinados que pueden ser modelos de intervención didáctica para introducir y establecer las leyes del movimiento uniformemente acelerado. Especial énfasis se ha puesto en la utilización de un plano inclinado sincrónico para establecer una de las formulaciones de Galileo acerca de las leyes que rigen este movimiento.

Palavras-chave: plano inclinado, movimiento uniformemente acelerado, Galileo.
\end{abstract}

In this work it is described some simple experimental settings in order to establish - in a didactical mode the laws of the uniform accelerated motion. Special emphasis has been done on the use of an isochronal incline for setting up one of the original Galileo' statements of the laws of this kind of movement.

Keywords: Galileo's incline, uniform accelerated motion.

\section{Introducción}

Los avances recientes en la didáctica de las ciencias experimentales han enfatizado la importancia de la experiencia (learning by doing) y el aprendizaje colaborativo (peer instruction) para obtener aprendizajes significativos. Todo ello dentro del paradigma constructivista. Aunque hay una amplia variedad de artículos didácticocientíficos que presentan los últimos avances en esta materia, permítasenos citar sólo algunos: los trabajos de McDermont et al. [1] y el texto de Viennot [2].

Ausubel $[3,4]$ dentro de su propuesta de aprendizaje significativo ha señalado la importancia de lo que el llama "organizadores previos" (advance organizers" Estas acciones - que pueden ser de diferente naturaleza - son las iniciales para construir (y/o reconstruir) sobre ellas el proceso de aprendizaje significativo. Las actividades que proponemos en esta nota, pueden ser entendidas como una de las acciones del tipo experimental que puede utilizarse con esta finalidad. Sin limitar la descripción de estos organizadores previos, otras actividades que pueden caer en esta categoría son: hechos históricos (científicos o no), experimentos pensados, la simple observación de fenómenos naturales, la aparente paradoja de algunas situaciones teóricas o experimentales, una noticia periodística o aún situaciones humorísticas o de historietas.

No hay que olvidar que - después de todo - lo que

\footnotetext{
${ }^{1}$ E-mail: cworner@ucv.cl.

${ }^{2}$ Esta caracterización se traduce, con frecuencia, como "analizadores de avance".
}

realmente importa como aprendizaje significativo es la existencia de leyes en los procesos naturales como lo describe bellamente el mismo Galileo, "(la ciencia)... è scritto in questo grandissimo libro che continuamente ci sta aperto. . Egli èscritto in lengua matematica" [5].

El instructor que enseña física debe entonces elegir cuidadosamente los instrumentos didácticos experimentales que puede (y debe) utilizar.

En la perspectiva de ampliar estos recursos para la enseñanza de la cinemática, específicamente el movimiento uniformemente acelerado, se describirán en la presente nota algunos usos sencillos del plano inclinado como actividad experimental. La característica principal de los aparatos y métodos que se detallarán es su simpleza conceptual y práctica. No es necesario utilizar relojes (en el sentido convencional) ni patrones de distancia (metros), sólo relojes "naturales" y razones de distancias.

Recientemente Soares y Borges [6] han comparado la realización del experimento del plano inclinado con métodos manuales (regla graduada y cronómetro) y con métodos digitales de adquisición de datos. Claramente ellos abogan por el uso de los métodos digitales. En sus propias palabras: "Com a realização da experiência utilizando coleta automática de dados e a construção de tabelas e gráficos usando o computador, o que tornou mais fácil e rápido fazer as medidas e construir os gráficos, possibilitou a realização desta atividade em 
um menor tempo". Sin desconocer el avance que significa el uso de los métodos automáticos de recolección de datos, la hipótesis del presente trabajo es que es posible realizar el experimento del plano inclinado aún sin usar relojes ni reglas graduadas. Es decir se trata de tener una experiencia didáctica simple con la menor cantidad de instrumentos de medida.

Por otra parte, también nuestra propuesta toca uno de los experimentos más famosos en la historia de la ciencia. El uso de los experimentos "históricos" en educación científica ha sido recientemente tratado en un trabajo de Chang [7]. Este autor enuncia una tipología de experimentos históricos, clasificándolos en i) réplicas históricas (estrictamente), ii) réplicas físicas (una recreación con modificaciones del original) y iii) extensiones a experimentos históricos. En nuestra propuesta tratamos de mostrar una aplicación didáctica, utilizando un experimento histórico

Un tercer aspecto a considerar es el resultado del aprendizaje que supuestamente, provoca el experimento. Freire y Moreira [8] han estudiado la evaluación de la enseñanza experimental en el laboratorio, señalando que "...muitas vezes os alunos não são capazes de identificar esses aspectos que poderiam caracterizados como constituintes da "estrutura" do experiment". Una explicación factible para esta percepción es que el excesivo uso de aparatos y/o cajas negras, desvirtúa la simpleza intelectual el tema que se desea exponer a los alumnos.

Los experimentos de Galileo con el plano inclinado son la inspiración del trabajo que se expone a continuación. Galileo [9] fue quien primero encontró las leyes del movimiento uniformemente acelerado con sus geniales experiencias usando un plano inclinado. Puede leerse una reproducción reciente de estos experimentos en un contexto didáctico, en los trabajos de Riess et al. [10] y Straulino [11]. También en el mismo espíritu han aparecido otros trabajos didácticos recientes basados en aspectos de la mecánica galileana [12-15]. De particular interés es la propuesta de De Hosson [16] de una reconstrucción dialógica - a partir de lo Diálogos de Galileo- de una propuesta didáctica relativa a sistemas en referencia con movimiento relativo entre ellos.

Así, el propósito de este artículo es mostrar cómo una adecuada reconstrucción de un experimento de origen histórico, con instrumental de uso nocontemporáneo (o no convencional), permite elaborar una ingeniería apropiada producir aprendizajes significativos en los estudiantes.

El movimiento de un móvil que desliza sin roce sobre un plano inclinado es uniformemente acelerado. También lo es el de una esfera (u otro objeto rodante) que rueda sin deslizar sobre el plano (que debe ser, en este caso, necesariamente rugoso). En esta última situación, con la advertencia que hay que tener debida cuenta del movimiento de rotación del objeto que rueda (véase cualquier texto de física universitaria introductorio, p. ej. Halliday et al. [17]). Sin embargo, como lo advierte Crawford [15] hay que considerar planos inclinados con pendiente "suave" como se discutirá al final de este trabajo.

Para fijar ideas (el lector puede hacer alguna otra elección dependiendo del nivel en que está enseñando) se propondrá obtener la ley que relaciona distancias y tiempos en el movimiento uniformemente acelerado expresada como: "Partiendo del reposo, las distancias parciales recorridas en tiempos iguales son entre sí como la sucesión de números enteros impares". Esto ahorra las discusiones habituales sobre las "fórmulas" y evita usar conceptos de difícil asimilación en un primer aprendizaje como los "segundos al cuadrado".

Hay que tener también presente que el movimiento de un móvil sobre el plano inclinado descubre preconceptos en los estudiantes [19]. Estos preconceptos deben ser considerados al realizar una intervención didáctica como la que se propone en el presente artículo.

\section{Planos inclinados didácticos}

Básicamente, la cinemática discurre en la medición de tiempos y distancias. De dichas mediciones es posible inferir leyes que resumen los resultados obtenidos de la experiencia. Al dejar rodar una esfera por un plano inclinado, las distancias recorridas pueden ser marcadas y medidas sobre el plano. El problema mayor consiste en la medición de los tiempos transcurridos.

Examinaremos algunos intentos didácticos obviar el uso del cronómetro. Para ello, Packard (citado por Greenslade [20] ) propone transformar el problema unidimensional en un problema bidimensional (véase Fig.1). Si lanzamos la esfera con una adecuada velocidad en el dirección horizontal, ésta describirá una parábola sobre la superficie del plano inclinado. De hecho, esto reproduce el conocido caso del movimiento parabólico pero con una aceleración ralentizada en la dirección del plano inclinado, $a=\gamma g \operatorname{sen} \theta$, donde $\gamma$ es un factor geométrico, $\theta$ es el ángulo que forma el plano inclinado con la horizontal y $g$ es la aceleración de gravedad.

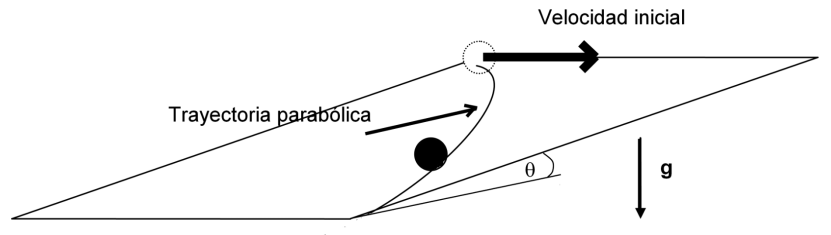

Figura 1 - Plano inclinado de Packard.

Si se dispone papel carbónico (llamado también papel calco) sobre la superficie del plano inclinado cubriendo un papel en blanco, se observará la traza del

\footnotetext{
${ }^{3}$ Es interesante notar que este experimento con el plano inclinado ha sido destacado como uno de los diez experimentos más bellos de la física de todos los tiempos http://physicsworld.com/cws/article/print/9746 (capturado, Enero 2012).
} 
movimiento parabólico de la esfera. La componente horizontal del movimiento es rectilíneo uniforme de tal modo que su traza horizontal se puede utilizar como reloj. Se divide el eje en porciones iguales y se leen los correspondientes valores sobre el eje del plano inclinado. Esto entrega valores de los espacios recorridos en intervalos de tiempos iguales y se puede descubrir que las distancias recorridas en tiempos iguales son entre si como los número naturales impares.

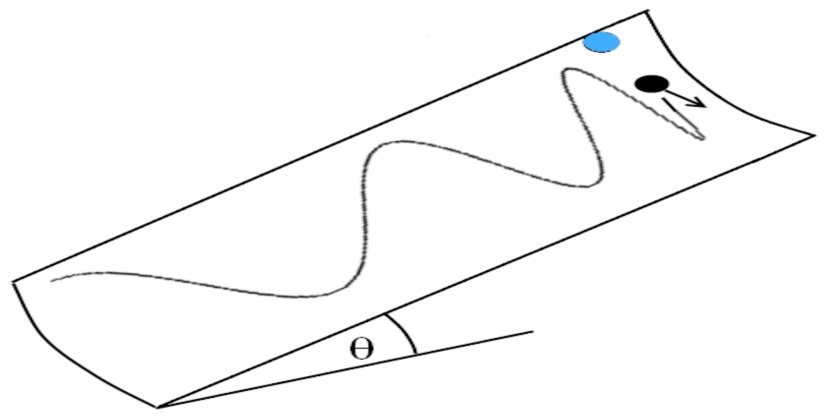

Figura 2 - Aparato de Duff.

Con similar ingenio, Duff (citado por Greenslade [21]), propone el uso de un plano inclinado "cóncavo" (ver Fig. 2). Allí, la forma de la trayectoria se puede visualizar poniendo polvo de tiza sobre la superficie. De nuevo, el movimiento se compone de un movimiento uniformemente acelerado (que se desea estudiar) y un movimiento oscilatorio que se utilizará como reloj. En este caso los tiempos se pueden medir con la suposición que el movimiento vibracional tiene período constante (ver la advertencia detallada en la sección final de esta nota).

Como lo ha hecho notar Reiss et al. [10] en el "Museum Boerhaave" (Leida, Holanda), hay un ingenioso aparato cuya construcción se atribuye a Jan van Musschenbroek que pre-fija una trayectoria parabólica (marcada por anillos por los que debe pasar una esfera) sobre un plano vertical (ver Fig. 3). Propiamente no es un plano inclinado, pero permite establecer las leyes del movimiento uniformemente acelerado de acuerdo a un procedimiento muy similar al propuesto por Packard.

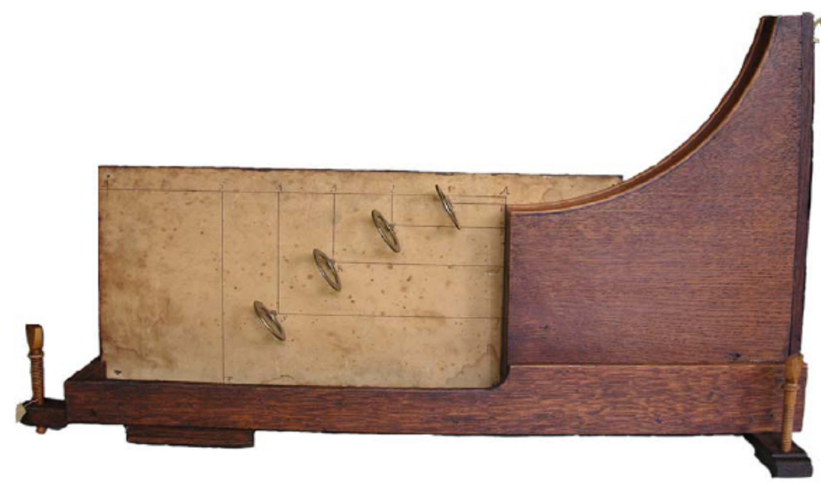

Figura 3 - Aparato de Musschenbroek (circa 1700).

\section{Plano inclinado sincrónico}

Los anteriores ejemplos extienden el problema del plano inclinado a un problema de dos dimensiones e implícitamente usan el "principio de independencia de los movimientos" adosando a nuestro experimento unidimensional un segundo movimiento ortogonal con rapidez uniforme que nos permite determinar los tiempos.

Distinto en su naturaleza es la aproximación experimental que se describirá en este párrafo. Al sentido de la vista, se agregará ahora la percepción del sonido como lo describe Drake [22] en su análisis de la obra de Galileo. También recientemente se ha hecho notar la influencia de la música en el desarrollo de la física, comentado la influencia de Vicenzo Galileo en la obra de su hijo, Galileo Galilei [23]. Hay que hacer notar que no hay evidencia histórica que Galileo haya efectivamente realizado este experimento. Sin embargo, hay una hermosa reproducción tardía de un plano inclinado con sensores acústicos (campanas) que data de principios del siglo XIX en el Museo Galileo en Florencia [24], (ver Fig. 4).

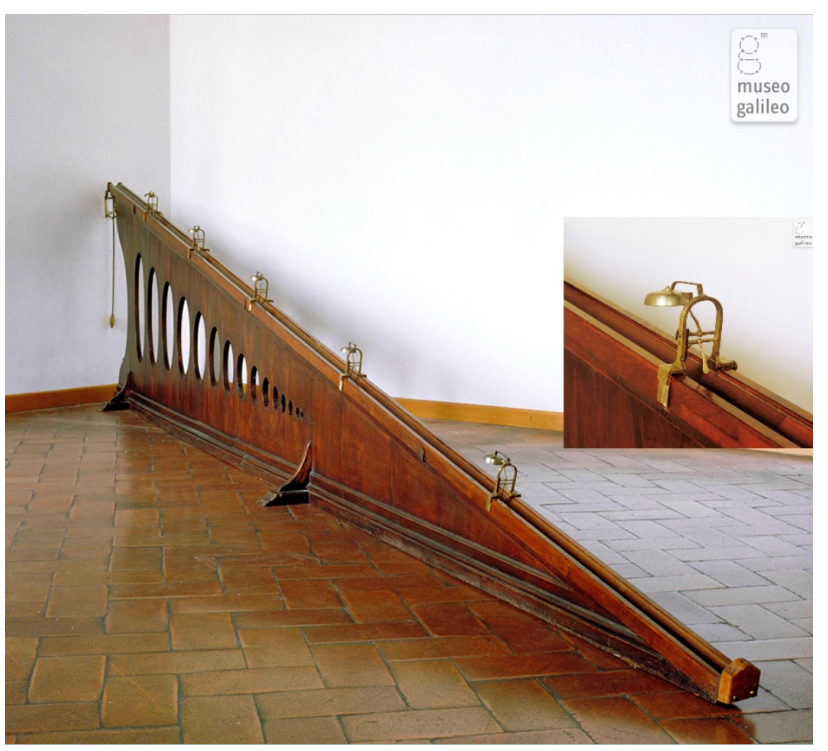

Figura 4 - Plano inclinado con "campanelle" (Museo Galileo, Florencia).

En este espíritu, en el presente trabajo, se utiliza un plano inclinado en que -a partir del extremo superiorse marcan distancias (por ejemplo, introduciendo un tornillo en una tabla de madera) siguiendo la serie de los número impares: 1, 3, 5, etc. (ver Fig. 5 y 6 ). De este modo, al hacer rodar una esfera desde la partida, se escuchará un golpeteo sincrónico al trasladarse sobre el plano inclinado. En el presente caso, el largo total del plano inclinado (una simple tabla acanalada) es de $240 \mathrm{~cm}$. La distancia unidad es de $8 \mathrm{~cm}$. Típicamente cada experimento demora algunos segundos. Es posible obtener distintas pendientes (y por lo tanto, distintas aceleraciones) simplemente soportando un extremo de riel con uno o más textos. La percepción del ritmo mu- 
sical en este tipo de experimentos, está explicitada en el ya citado trabajo de Crawford [18].

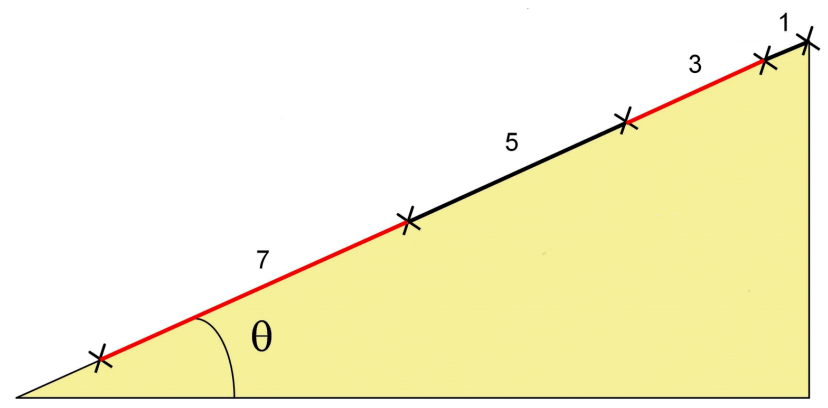

Figura 5 - Plano inclinado sincrónico (los números representan las distancias entre la marcas señaladas, en unidades arbitrarias).

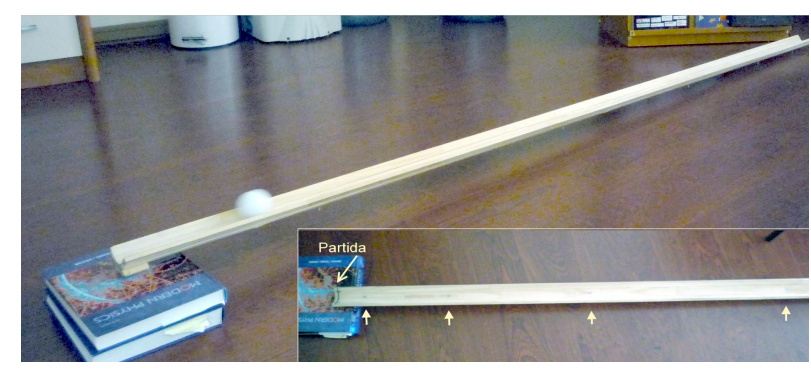

Figura 6 - Plano inclinado sincrónico. En el inserto se señalan las posiciones de los tornillos.

El problema didáctico es ¿cómo - partir de este simple experimento- se puede inducir una apreciación más científica del movimiento sobre el plano inclinado?. Para esto, se puede diseñar una secuencia didáctica como se muestra en el esquema de la Fig. 7 que se autoexplica. La finalidad es describir experimentalmente lo enunciado por Galileo: "...Si en tiempos iguales, tomados sucesivamente desde el primer instante o comienzo del movimiento, tales como AD, DE, EF, FG, se recorrieren los espacios HL, LM, MN, NI, estos espacios estarán entre sí, como los números impares 1,3 , 7;..." (Galileo, traducido por San Román, 1945). Para mejor comprensión del enunciado de Galileo, véase el diagrama original del texto de Galileo [9] reproducido aquí como Fig. 8.

Esta verbalización de la ley de los espacios en el movimiento uniformemente acelerado no es sino una formulación equivalente al conocido resultado para el movimiento uniformemente acelerado que establece que las distancias totales recorridas, partiendo del reposo, son proporcionales a los cuadrados de los tiempos, como también describió Galileo en la ya citada obra. En opinión de quien escribe, el primer enunciado es conceptualmente más simple que el segundo y puede ser usado como una definición alternativa del movimiento uniformemente acelerado.

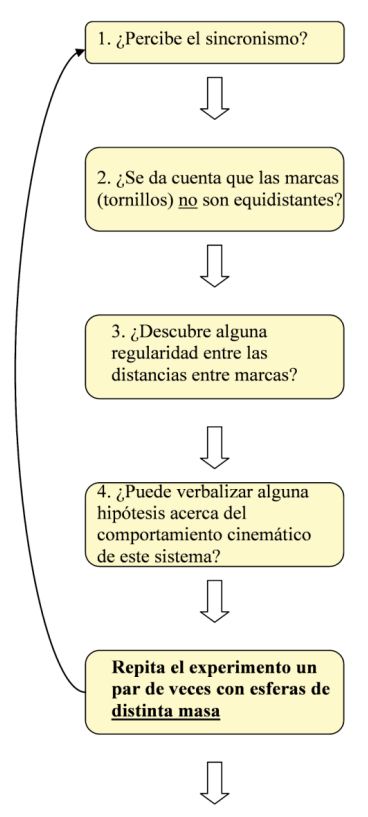

(sigue en la otra columna)

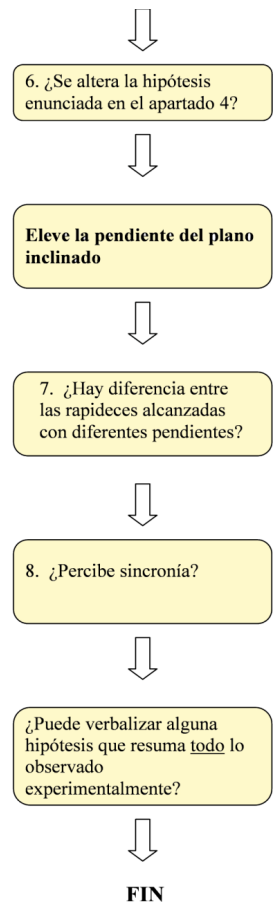

Figura 7 - Esquema tentativo para una intervención didáctica.

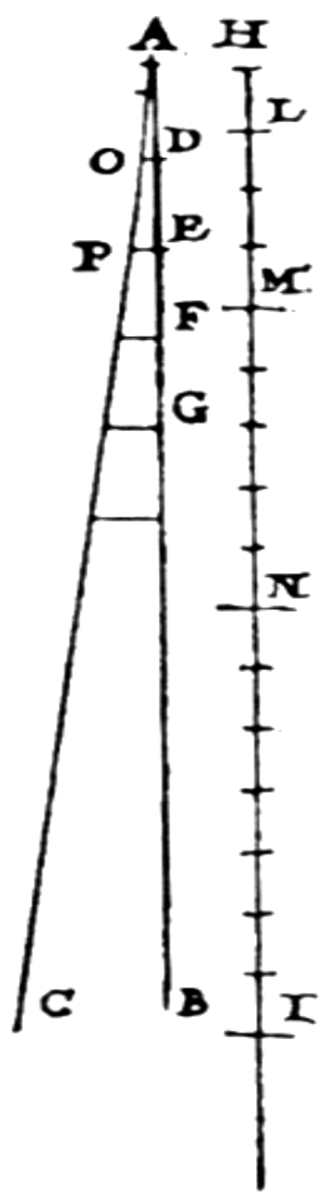

Figura 8 - Esquema de Galileo para explicar el enunciado de la ley de los números impares. 


\section{Discusión}

Debe hacerse notar que todos estos aparatos son de construcción sencilla y robusta. De hecho pueden armarse in situ. También poseen la característica común de no depender de otros instrumentos de medida como metros y relojes. El tiempo se mide en unidades arbitrarias determinadas por el período de sonido de los choques entre obstáculo y obstáculo y las distancias pueden determinarse usando algún patrón arbitrario. Dicho en otros términos, la descripción de los problemas se establece en términos de proporciones y eso refuerza la idea que las leyes de la naturaleza no dependen de algún particular sistema de pesas y medidas. Esto es un ejemplo de escalado (scaling) $[12,13,15]$ muy usado en la literatura científica contemporánea y además nos remite a la época y los métodos que usó Galileo en sus descubrimientos. Dicho sea de paso, Galileo no utilizaba el álgebra es sus desarrollos; sólo la clásica geometría griega.

Finalmente, dos observaciones:

Usualmente se dice que aumentando la pendiente y mediante un paso al límite (algo así como un "experimento pensado"), se puede mostrar que la caída libre es un movimiento uniformemente acelerado. Sin embargo se ha demostrado que tal paso es empíricamente incorrecto ya que al aumentar el ángulo del plano inclinado, la simple rodadura de la esfera se convierte - a lo menos parcialmente- en deslizamiento [18].

El uso del plano de Duff agrega una complicación al desarrollo lógico del experimento. El movimiento oscilatorio de la esfera (que sirve de "reloj" en el experimento) no tiene un simple análisis ya que necesariamente si la esfera rueda sin deslizar requiere la introducción del momento de inercia como parámetro. En este caso, se puede demostrar [25] que el movimiento es armónico simple y que el período de oscilación para amplitudes pequeñas (como las de nuestro experimento) está dado por

$$
T=\sqrt{2 \pi\left[\frac{(R-r)(1+\gamma)}{g}\right]},
$$

donde $R$, es el radio de la sección circular, $r$ es el radio de la esfera, y $\gamma$ es un factor geométrico que en el caso de una esfera es igual a $2 / 5$.

Resumiendo, con las debidas precauciones (preconceptos, rodadura y deslizamiento y período para el caso del plano de Duff) esta propuesta debiera transformarse en un instrumento útil para el profesor de mecánica elemental. Se une así la simplicidad, los aspectos históricos y la ingeniería didáctica para el aprendizaje de la cinemática del movimiento uniformemente acelerado.

\section{Agradecimientos}

Se agradece la colaboración y esfuerzo de los estudiantes y ahora titulados, O. Carriel, R. Riquelme y B. Merello. También las discusiones técnicas con mi colega
A. Romero. Este trabajo forma parte de un proyecto DI-PUCV-037.275/2012.

\section{Referencias}

[1] L.C. McDermont, P.L.R. Heron, R.S. Shaffer y M.R. Stetzere, American Journal of Physics 74, 763 (2006).

[2] L. Viennot, Teaching Physics (Kluwer Academic Publishers, Dordrecht, 2003).

[3] D.P. Ausubel, Journal of Educational Psychology 51, 267 (1960)

[4] D. Ausubel, Review of Educational Research 48, 251 (1978).

[5] G. Galilei , Il Saggiatore, http://www.pelagus.org/ it/libri/IL_SAGGIATORE,_di_Galileo_Galilei_1. Iftm (capturado Enero, 2012).

[6] R.R. Soares y P.F. Borges, Revista Brasileira de Ensino de Física 32, 2501 (2010).

[7] H. Chang, Science \& Education 20, 317 (2011).

[8] M.A. Freire Passos y M.A. Moreira, Revista Brasileira de Física 12, 375 (1982).

[9] G. Galileo, Discorsi e Demostrazione Mathematiche in torno a Due Nuove Scienza Attenenti alla Mechanica Es i Movimiento Locale, (Elzevier, Leyden, 1632). Hay una versión en español: J. San Román-Vicente (con notas de T. Isnardi), Diálogos Acerca de Dos Nuevas Ciencias (Losada, Buenos Aires, 1945).

[10] F. Riess, P. Heering y D. Nawrath, Reconstructing Galileo's Plane Experiments for Teaching Purposes, www.ihpst2005.leeds.ac.uk/papers/Riess_ Heering_Nawrath.pdf (capturado en Enero 2012).

[11] S. Straulino, Physics Education 42, 316 (2008).

[12] C.H. Wörner y G. Iommi Amunátegui, European Journal of Physics 28, 643 (2007).

[13] C.H. Wörner, Physics Education 42, 437 (2007).

[14] S. Straulino, A.M. Gambi y A. Riglini, American Journal of Physics 79, 32 (2011).

[15] S. Straulino, Physics Education 46, 206 (2011).

[16] C. de Hosson, Enseñanza de las Ciencias 29, 115 (2011).

[17] D. Halliday, R. Resnick y K.S. Krane, Physics (J. Wiley \& Sons, New York, 1992), 4th ed.

[18] F.S. Crawford, American Journal of Physics 64, 541 (1996).

[19] D. Rohder, Memory \& Cognition 31, 816 (2003).

[20] T.B. Greenslade, The Physics Teacher 34, 156 (1996).

[21] T.B. Greenslade, The Physics Teacher 41, 170 (2003).

[22] S. Drake, Scientific American 232, 98 (1975).

[23] I. Caleon y S. Ramanathan, Science \& Education 17, 449 (2008).

[24] Museo Galileo, Florencia, http://catalogo. museogalileo.it/oggetto/PianoInclinato.html (capturado, Enero 2012).

[25] D.T. Gillespie, American Journal of Physics 52, 180 (1984). 